\title{
Measuring response inhibition with a continuous inhibitory-control task
}

\author{
Christina Meier $^{1} \cdot$ Stephen E. G. Lea ${ }^{1} \cdot \operatorname{lan}$ P. L. McLaren ${ }^{1}$ \\ Published online: 29 January 2020 \\ (C) The Psychonomic Society, Inc. 2020
}

\begin{abstract}
Inhibitory control enables subjects to quickly react to unexpected changes in external demands. In humans, this kind of behavioral flexibility is often used as an indicator of an individual's executive functions, and more and more research has emerged to investigate this link in nonhuman animals as well. Here we explored the value of a recently developed continuous inhibitorycontrol task in assessing inhibitory-control capacities in animals. Pigeons completed a response-inhibition task that required them to adjust their movement in space in pursuit of a reward across changing target locations. Inhibition was measured in terms of movement trajectory (path taken toward the correct location for trials in which the target location did and did not change) and velocity (both before and after correcting the trajectory toward the changed location). Although the observed velocities did not follow any of our predictions in a clear way, the pigeons' movement trajectories did prove to be a good indicator of inhibitory control, showing that pigeons, though limited in their capacities relative to the sophisticated control strategies expressed by humans, are capable of exerting some forms of inhibitory control. These results strengthen the role of this paradigm as a valuable tool for evaluating inhibitory-control abilities across the animal kingdom.
\end{abstract}

Keywords Inhibition $\cdot$ Pigeon $\cdot$ Inhibitory control $\cdot$ Response inhibition

Humans, and most nonhuman animals, are able to quickly adapt their behavior to unexpectedly changing external demands - for example, by inhibiting inappropriate or no longer relevant behavior, or adjusting an action that has already been initiated (e.g., Ardila, 2008; Chan, Shum, Toulopoulou, \& Chen, 2008; Jurado \& Rosselli, 2007; Suchy, 2009). Such mental flexibility, more specifically referred to as inhibitory control, has long been considered to be an indicator that an individual possesses higher-order cognitive control abilities (e.g., Coutlee \& Huettel, 2012; Kabadayi, Bobrowicz, \& Osvath, 2018), as it requires a chain of cognitive operations (including detecting the currently relevant response requirements, selecting and implementing the appropriate response and simultaneously inhibiting alternative response options; Verbruggen \& Logan, 2015; Verbruggen, McLaren,

Electronic supplementary material The online version of this article (https://doi.org/10.3758/s13420-019-00403-7) contains supplementary material, which is available to authorized users.

Christina Meier

meierchristina@aol.com

1 University of Exeter, Exeter, UK
$\&$ Chambers, 2014). This assumption implies that only those species that possess a high level of cognitive control, such as humans, apes, and other big-brained mammals and birds, should be capable of inhibitory control. However, the emergence of studies providing evidence for inhibitory control across a broad range of species [e.g., dogs (Canis familiaris): Fagnani, Barrera, Carballo, \& Bentosela, 2016; MarshallPescini, Virányi, \& Range, 2015; Vernouillet, Stiles, McCausland, \& Kelly, 2018; sheep (Ovis aries): Knolle, Goncalves, Davies, Duff, \& Morton, 2019; Knolle, McBride, Stewart, Goncalves, \& Morton, 2017; rats (Rattus norvegicus): Beuk, Beninger, \& Paré, 2014; Eagle \& Robbins, 2003; pheasants (Phasianus colchicus): Meier et al., 2017; guppies (Poecilia reticulata): Lucon-Xiccato, Gatto, \& Bisazza, 2017; Santacà, Busatta, Savașç1, Lucon-Xiccato, \& Bisazza, 2019] is casting doubt on this all-or-nothing approach implied in the earlier work.

Recently, attention has shifted from merely investigating whether members of a given species possess the ability to inhibit their actions when necessary to establishing a more complete picture of varying degrees of the ability to implement inhibitory control, both across and within species. A promising paradigm in this regard is the continuous response-inhibition task, which was first developed by 
Verbruggen and McLaren (2017) to assess the development of inhibitory control during human childhood, and was later adapted by Meier et al. (2017) for use with animals.

In the broadest terms, the paradigm required subjects to leave a start location (in the human computer version, this was the location from which a mouse cursor had to be moved; in the animal version, it was the location from which the subject entered an experimental arena) and move toward a goal to obtain a reward (see Fig. 1 for a two-dimensional schematic of the setup). In the majority of trials, the goal was at a fixed location relative to the start location, and the subject was encouraged to go from the start to the goal as quickly as possible (trials of this nature will henceforth be referred to as "go trials," and the location that has to be reached to obtain the reward will be called the "go location"). In a minority of trials, however, shortly after the subject had left the start location and was on the way toward the goal, the location of the goal suddenly changed from its previously designated location to a new one (trials of this type will henceforth be referred to as "change trials," and the location that has to be reached to obtain the reward will be called the "change location"). To complete a change trial and obtain a reward, the subject had to alter the initial course and pursue the goal at its new location (see Fig. 1).

The advantage of this continuous response-inhibition task for investigating individual inhibitory control is the wide variety of

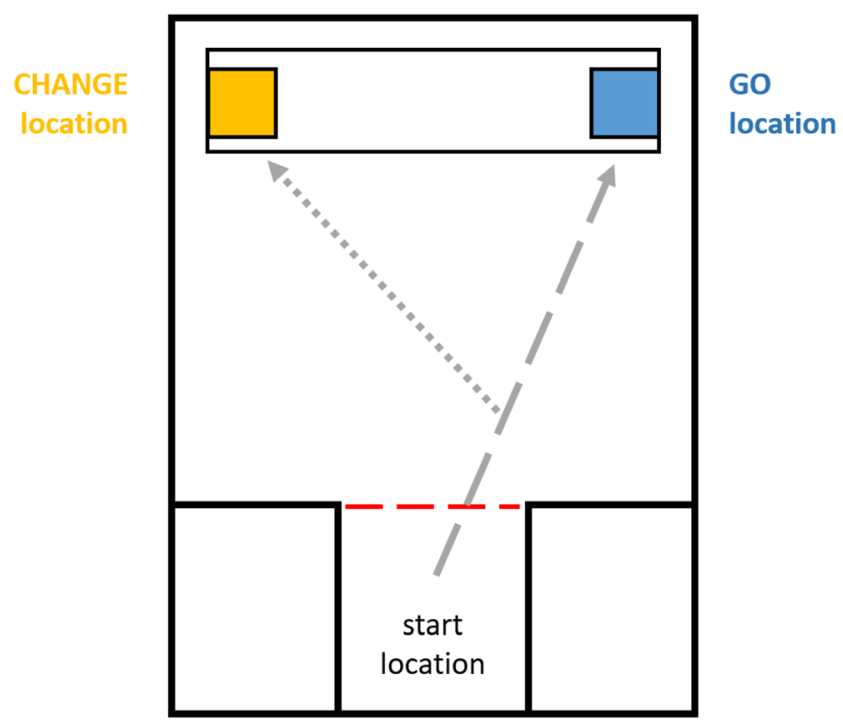

Fig. 1 Diagram of the continuous response-inhibition task for animals (adapted from Meier et al., 2017). A subject starts a trial by leaving the start location, shown at the bottom of the image. In the majority of trials, the subject has to pursue a path, indicated by the dashed gray arrow, toward the go location (illustrated on the right) to obtain a reward. On a minority of trials, however, the target location visibly changes from the go location to the change location (illustrated on the left) as the subject leaves the start location (this moment is indicated by crossing the horizontal dashed line across the start location exit); to obtain the reward, the subject has to then alter its path toward the change location, indicated by the dotted gray arrow. dependent variables that can be assessed in order to obtain a detailed picture of a subject's cognitive abilities and strategies.

Verbruggen and McLaren (2017) employed their human version to determine the age at which children begin to show evidence of proactive inhibitory control in performing "mental goal adjustments" between two trials (Bissett \& Logan, 2011, 2012; Verbruggen, Schneider, \& Logan, 2008); according to this account, on each trial, the subject is faced with a conflict between two mental goals - on the one hand, there is the goal to quickly perform the go response (i.e., to reach the go location), and on the other hand, there is the goal to respond accurately to any sudden change of the response requirements (i.e., an unexpected change of the goal location), which requires the subject to divert attention from the primary goal. To respond both quickly and accurately, it is assumed that humans continuously calibrate the balance between focusing mental resources on one goal over the other (cf. Elchlepp, Lavric, Chambers, \& Verbruggen, 2016).

Adjusting the balance in favor of the mental goal to respond quickly might increase the likelihood of executing the "go" response not only when it is appropriate, but also when it is inappropriate (i.e., on change trials), whereas adjustments toward the mental goal to detect a change might facilitate the inhibition of that "go" response when necessary but will also lead to slower response execution when the "go" response is indeed required. It is assumed that humans adjust the balance in favor of change detection after experiencing a trial in which the "go" response had to be inhibited, and in favor of the "go" response after a go trial. Consequently, such mental goal adjustments are expressed in the way in which the cognitive control afforded in one trial influences performance on the following trial: On a go trial following a change trial, latencies to reach the goal would be slower than on a go trial preceded by another go trial. Furthermore, Verbruggen and McLaren (2017) argued that a subject's path toward the go location would be less direct and more skewed toward the change location on trials following a change trial than on trials following a go trial. Figure 2A, left column, shows an illustration of the expected path for each trial type if the subject performs mental goal adjustments between trials. In addition to trajectories, the logic of Verbruggen and McLaren implies that such mental processes would also manifest in the speed with which the subject pursues its goal, perhaps speeding up to complete the "go" response quickly, or slowing down to be able to respond to the sudden change of location. A subject's velocity during a given trial can thus give further valuable information about its cognitive strategy: It might be expected that subjects would enter the arena at a slower pace on go trials following a change trial than on those following a go trial, although they might speed up once the change has occurred (or once it becomes unlikely to occur). The predicted average velocity of subjects from this perspective is shown in Fig. 2A, right column. 
_ Go trial (previous trial was a Go trial)

- - - Go trial (previous trial was a Change trial)

Change trial

a) Proactive Inhibitory Control
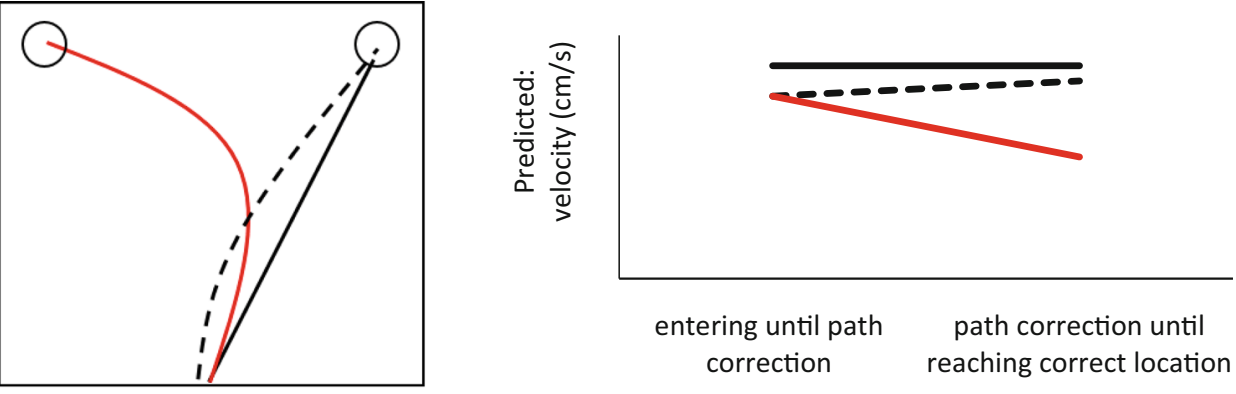

b) Reactive Inhibitory Control
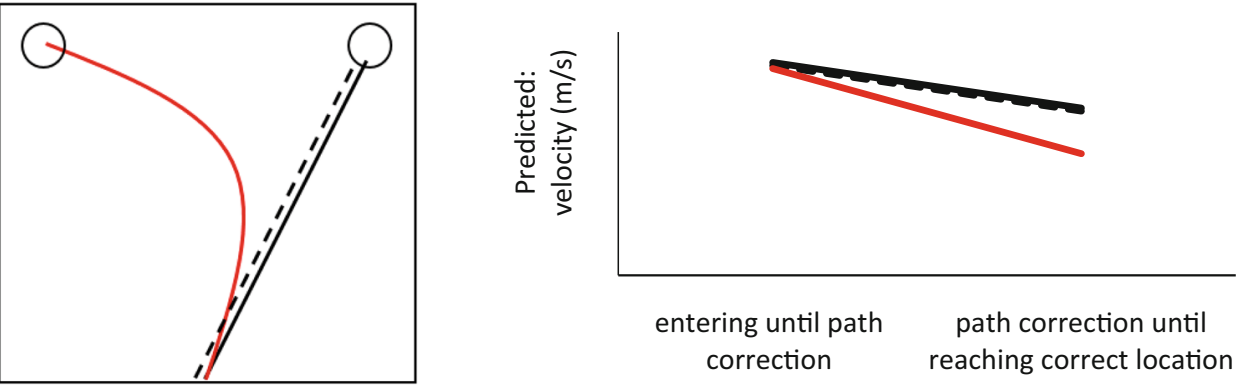

c) Response Repetition
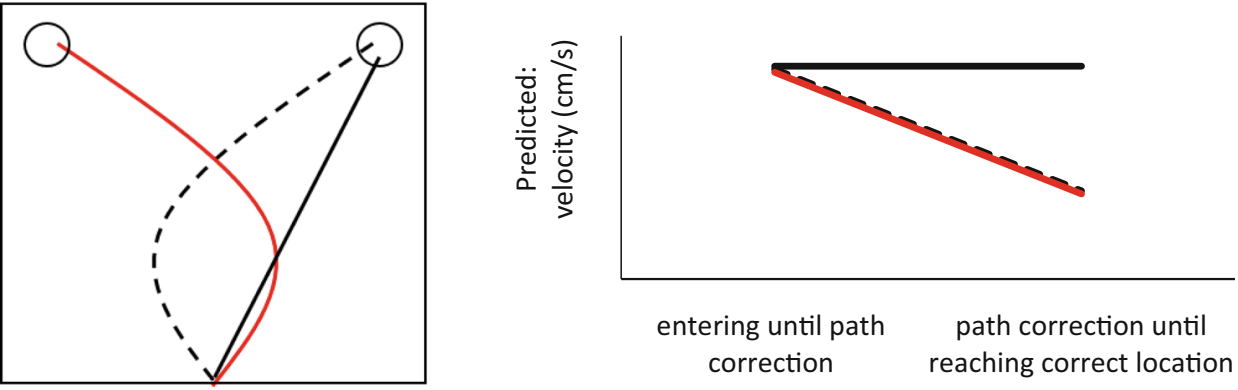

d) No Discrimination

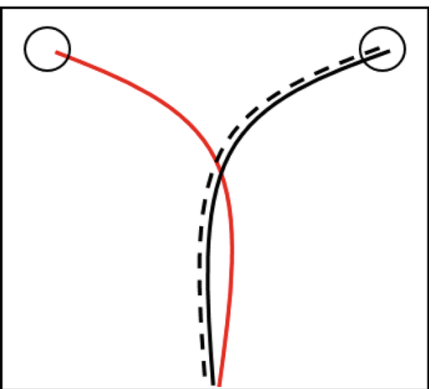

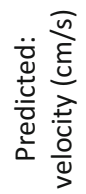

Fig. 2 Left column: Expected path trajectories in go trials (following a go or following a change trial) and change trials if behavior is governed by (A) proactive mental goal adjustments between trials, (B) reactive inhibition in response to immediate changes, $(\mathrm{C})$ a tendency to approach the

In their avian version of the continuous response-inhibition task, Meier et al. (2017) demonstrated that pheasants were

able to adjust their paths flexibly from one goal to another. Their paradigm was not set up, however, to capture the subtle 
influences of response requirements experienced on previous trials, thus leaving an open question as to whether such intertrial effects are evidence of mental goal adjustments and the proactive inhibitory control they imply, or whether less cognitively demanding strategies might have similar effects on performance.

First, it is important to differentiate the proactive inhibitory process described above from reactive inhibition (cf. Meier, Lea, \& McLaren, 2018), which might lead to a quite different pattern of responding. If subjects relied on reactive control, each trial for a given subject would start in much the same way (i.e., there would be no effect of one trial on another). Thus, a subject might begin by rapidly approaching one location (typically the initially indicated go location), but at some point, this initial trajectory will be brought under control of the information present on that trial. During a go trial, regardless of the response requirements of the previous trial, because there is no change in the presented information, the subject would continue on its original trajectory. During change trials, however, the subject would subsequently correct the trajectory in response to the goal change from the go to the change location. Figure 2B, left column, shows an illustration of the expected path for each trial type if the subject pursues a strategy of response repetition. Contrary to the proactive-inhibition approach, subjects would not be expected to alter their velocity on the basis of the previous trial contingencies if relying on response repetition; they might show the same speed upon entering the arena in all trial types. However, they would be expected to gradually slow down when the subject's movements change from an initially fast launch toward the initial target location, akin to a ballistic phase, toward a controlled phase that is slower. The controlled phase involves the use of visual feedback to ensure a continuous reduction of the subject's distance from the goal location; thus, during trials that require greater adjustments to one's trajectory - that is, change trials - the decrease in velocity would be expected to be greater than that during go trials. The predicted average velocity of subjects pursuing this strategy is shown in Fig. 2B, right column.

Second, differences in trajectory patterns could emerge if subjects had a tendency to repeat a previously rewarded response, that is, a tendency to return to the most recently rewarded location, as has been demonstrated to occur early in behavioural training (Morgan, 1974). In such a case, in go trials following a change trial, the paths taken from the start location to the goal would show an initial approach toward the change location, just as the trajectories in change trials following a go trial would initially be biased toward the go location, before being adjusted toward the correct location. A straight and direct path toward the go location would only be observed in go trials following another go trial. Figure $2 \mathrm{C}$, left column, shows an illustration of the expected path for each trial type if the subject pursues a strategy of response repetition. As in the previous approach, subjects would be expected to show the same speed upon entering the arena in all trial types. Contrary to the reactive-inhibition account, however, they might slow down not only in change trials but also in go trials following a change trial, as in both trial types it would become necessary to correct the initial trajectory. The predicted average velocities of subjects pursuing this strategy are shown in Fig. 2C, right column.

Finally, subjects might hesitate to approach either the go or the change location - or be unable to discriminate between the two - until the change did (or did not) occur and the correct location was unambiguously indicated. In this case, subjects might try to keep an equal distance from both locations as they leave the start location, so that the resulting path would lead up in a straight line between the two options before branching off toward the correct location at a sharp angle. Figure 2D, left column, shows an illustration of the expected paths for each trial type if the subject did not discriminate between the two goal options. The same pattern of average path trajectories would be observed if the subject simply chose a first location at random and approached both locations in succession, regardless of the actual demands of the trial. Regarding predicted velocity, there should be no differences between trials, and subjects might proceed at the same speed from entering the arena to arriving at the correct location, or might speed up the closer they got to the see-saw. The predicted average velocities of subjects pursuing this strategy are shown in Fig. 2D, right column.

In the present study, we assessed which one, if any, of the above mechanisms most accurately describes the response patterns of pigeons (Columba livia), which can perform successfully in response-inhibition tasks despite being limited in their cognitive-control capacities (Lea, Chow, Meier, McLaren, \& Verbruggen, 2019; Meier et al., 2018). In both computerized response-inhibition tasks previously employed with pigeons, subjects showed a pattern of responding that was consistent with a popular model of reactive response inhibition, the independent horse-race model (cf. Verbruggen \& Logan, 2009): The pigeons were increasingly unable to correct their behavior as the time between the initial stimulus and the signal to inhibit an action decreased. We adapted Meier et al.'s (2017) paradigm in order to examine whether the performance of pigeons reflects

a) proactive inhibitory control (expressed through mental goal adjustments),

b) reactive inhibitory control,

c) a bias to approach the previously rewarded location, or

d) hesitation or inability to commit to one particular location.

Each of these mechanisms is expected to result in a distinct pattern of path trajectories toward the appropriate goal location and in different response-time patterns, shown in Fig. 2. 


\section{Method}

\section{Subjects}

A total of 49 adult pigeons were entered in this experiment; 38 of them completed the entire procedure. They had previous experience with computer-based experiments, in some cases in experiments that tested inhibitory control (Lea et al., 2019; Meier et al., 2018), but none in an apparatus resembling that used in the present experiment. The pigeons had been obtained as discards from local fanciers, so their ages were not known exactly. Their sexes were not determined. They were kept in the Psychology animal laboratory at the University of Exeter. For the duration of the experiment, they were housed together with other pigeons in two indoor aviaries (each approximately $2 \times 1 \times 2.5 \mathrm{~m}$ ); since most of them had plain white plumage, they were identified by colored leg rings. They were maintained at or above $80 \%$ of their free-feeding weight by controlled feeding after tests. Their weights at time of test ranged from 230 to $400 \mathrm{~g}$, corresponding to a variation of linear size of about $20 \%$, or $5 \mathrm{~cm}$, which is a small variation in size compared with the dimensions of the apparatus. On conclusion of the experiment, the pigeons were moved to a collective outdoor aviary with unlimited access to food. Each pigeon was tested in isolation. Inside the testing arena, the pigeons had no access to water or grit, but they received water and grit ad libitum in all aviaries and holding areas.

\section{Apparatus}

Figure $3 \mathrm{~A}$ is a scale plan of the experimental arena; for a 3-D sketch, see Fig. 3D. The arena was mounted onto a sheet of $135 \times 100 \mathrm{~cm}$ melamine-covered chipboard (Contiboard) with six pillars of planed softwood, each $10 \times 10 \times 45 \mathrm{~cm}$ in size. The outer walls of the arena were made of 6-mm plywood of $30 \mathrm{~cm}$ in height; the long walls were $124 \mathrm{~cm}$ long, and the short walls were $80 \mathrm{~cm}$ long. Because the poles were taller than the walls, a curtain made of blue cloth was drawn between the poles along the long walls (see Fig. 3B), to restrict the pigeons' vision to the inside of the arena. The arena that was accessible to the pigeons measured $82 \times 76 \mathrm{~cm}$. The pigeons entered the arena from one of the short walls via a 40-cm-long and 26-cm-wide runway corridor (see Fig. 3C). The corridor and the interior walls adjacent to it were made of 6-mm plywood and plastic-covered 25-mm chicken wire, enabling the pigeons to view the arena from inside the corridor. A see-saw apparatus was mounted along the opposite short wall of the arena. It consisted of a plywood beam $50 \mathrm{~cm}$ in length that was hinged on a bolt attached to a block of wood $10 \mathrm{~cm}$ off the ground. The block of wood was mounted onto a $60 \times 15 \times 2 \mathrm{~cm}$ board of wood. A $10 \times 10 \mathrm{~cm}$ white Perspex baseplate was screwed flat onto each end of the board to indicate the two food locations, the centers of which were $35 \mathrm{~cm}$ apart from each other (see Fig. 3B). A small plastic cup was attached to each end of the see-saw axis, cut to size so as to cover another plastic lid glued in place onto the baseplate underneath that served as a food well. The see-saw could be operated to cover one of the two food wells. In its default position, the see-saw covered the left (as seen from the corridor entrance) food well, exposing the right well. The beam rested on the armature of a solenoid integrated into the wooden block that held the see-saw hinge. The armature retracted when the solenoid was activated, allowing the seesaw to tip over to cover the right food well and expose the left well. Directly adjacent to the right side of the corridor exit on the inside of the testing area, an infrared LED diode was mounted $7 \mathrm{~cm}$ from the ground, which continuously generated an infrared beam. The beam was detected by an infraredsensitive lux meter mounted at the same height on the left side of the corridor exit. The lux meter recorded any changes in lux of the incoming infrared beam and submitted this information to an Arduino One microchip board; if there was an interruption in the light beam, the Arduino One board operated the solenoid attached to the see-saw. The arena was covered by a Plexiglas roof hinging onto one of the long walls. It opened upward to allow the experimenter to manually remove the pigeon from the arena. The runway corridor was covered by a scrap-board roof. A Kenvo HDV-601S video camera was mounted onto this roof, overlooking the testing arena, to film the trajectories of the participating birds.

\section{Procedure}

Before testing, all pigeons received a 15-min habituation session in which the see-saw was fixed in place in order to expose both food wells, which were both baited. One pigeon at a time was placed into the entrance corridor of the arena and was allowed to freely explore the testing arena and feed from the two food wells. A pigeon received up to three habituation sessions until it had fed from both food wells in the same session. If a pigeon had not visited both wells by the third session, it was excluded from any further test sessions. Following a successful visit to both food wells within 15 $\mathrm{min}$, the pigeon received one 5-min session in which, again, both food wells were accessible and baited. If the pigeon visited both wells within the 5-min interval, it was moved on to the test sessions. This 5-min feeding session was also repeated up to three times, until the pigeon had fed from both wells in the same session. As above, if a pigeon failed to do so within three sessions, it was excluded from any further test sessions. Thirty-eight of the 49 pigeons successfully completed the habituation sessions; the remaining 11 pigeons did not sufficiently explore the arena, either during the 15-min or the 5min sessions. We did not observe any visible preference for 

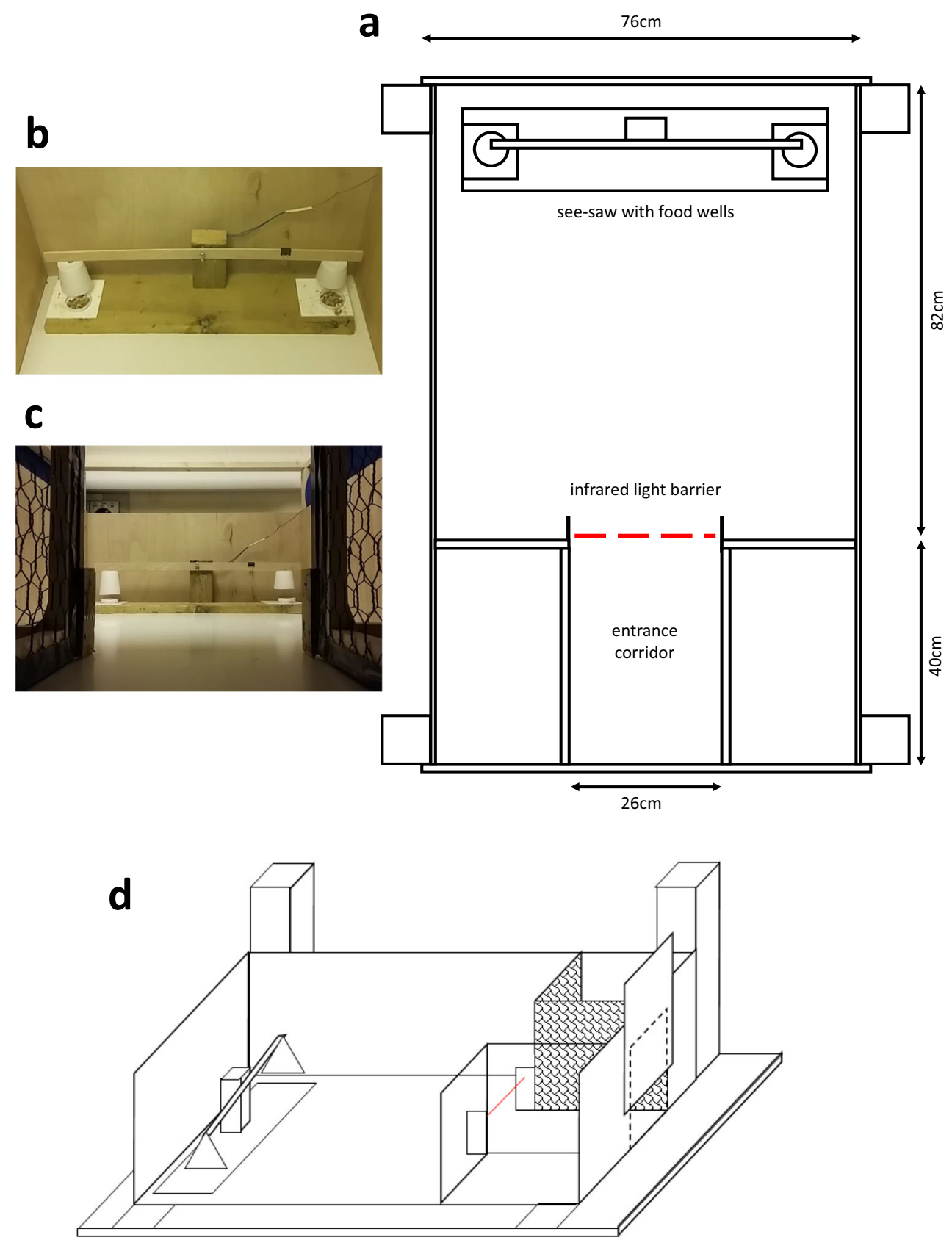

Fig. 3 (A) Top-down plan of the testing arena. (B) View from above, looking at the see-saw. Both food locations are uncovered in this picture. (C) The pigeon's view from inside the entrance corridor into the arena.

either location at the group level during the habituation phase (Supplementary Table 1, rightmost columns).

Test trials were administered in two sessions, presented on two consecutive days, each session consisting of four blocks of three trials, totaling 24 trials per pigeon. Within a session, the blocks were administered immediately one after the other, with a maximum of $5 \mathrm{~min}$ between trials to allow for a reset and cleaning of the arena. The first two trials in each block were go trials: The see-saw was fixed in order to expose the right food well (covering the left well), and a pigeon was
Both food locations are uncovered in this picture. (D) Design of the testing arena, shown from the side (not to scale; for the exact proportions, see panel A). See the text for details.

allowed to feed freely from that well after approaching it. The third trial in a block was a change trial: At the start of this trial, the see-saw was in the same position as in go trials, but it rested loosely on the solenoid armature, so that it tipped to the other side when the pigeon crossed the infrared light beam at the arena entrance and so operated the solenoid. In these trials, the pigeon was allowed to feed from the newly exposed left food well; the right well was covered after the see-saw had tipped over. The sequence of trials was the same for all subjects. 
A trial ended either once the pigeon had consumed all the food from the available food well or, if the pigeon failed to approach that well, after a maximum of $3 \mathrm{~min}$. In the latter case, the trial was repeated up to two more times until the pigeon approached the exposed food well; if it had not done so by the third trial repetition, the session was aborted. If this was the case, the pigeon repeated the entire block of three trials in its next session.

\section{Data collection}

Because in the very first trial, subjects would not yet have any concept of the task demands, it was excluded from the analyses. Thus, we analyzed responses for seven go trials preceded by a change trial, eight go trials preceded by a go trial, and eight change trials.

We determined the subjects' trajectories as they moved within the arena and toward the correct food location. Using the Open Source Physics Tracker video-tracking software (Brown, 2009), the coordinates of a pigeon's beak were extracted from the videos of each trial. The axes of coordinates were standardized so that the point of crossing the infrared beam coincided with the point of crossing the $x$-axis at ( $x$, 0 ); the available food location in go trials was located at (1, 1 ), and the available food location in change trials was located at $(-1,1)$. To account for individual differences in the latencies to reach the correct food location, the trajectories of each trial were standardized in a way similar to Vincentization (Genest, 1992; Ratcliff, 1979; Rouder \& Speckman, 2004; Vincent, 1912); that is, the value of the latency for a given trial was split into twenty 0.05 -quantile points; the coordinates at each quantile point were connected to create the trajectory for that trial. These were then averaged across the eight (or seven) trials of each trial type for each pigeon.

To obtain distinct data points that could be used to compare the trajectories between trials, we extracted an individual's trajectory-correction points for each of the three trial types from the trajectory data. The trajectorycorrection point is defined as the inflection point in a pigeon's trajectory. For change trials, this was the point at which the $x$-coordinate of the trajectory reached its final local maximum value before it continuously decreased. It indicates the moment at which the subject started approaching the (correct) change location. For go trials, it was the point at which the $x$-coordinate reached its final local minimum value before it continuously increased, indicating the moment at which the subject started to directly approach the (correct) go location.

In addition, we recorded the latency from releasing a pigeon into the entrance corridor until it reached the correct food well in each trial, to get an estimate of the overall time taken to complete the trials that required behavioral adjustments (i.e., change trials) as compared to the time taken to complete the trials in which no such adjustments had to be made (go trials). The conventional measure of response inhibition is the change-signal reaction time (Verbruggen \& Logan, 2009; Verbruggen \& McLaren, 2017) — that is, the latency between the onset of the signal to withhold the target response and the observable change in behavior. In our continuous response-inhibition task, this is equivalent to the time to reach the trajectory correction point, measured from the moment of crossing the infrared light beam. However, pure latency measures might be confounded in this task because of the simple fact that it might take more time to cover a longer distance (comparing, e.g., the assumed straight trajectory during go trials to the predicted curved path during change trials). A more accurate measure of inhibition processes might therefore be the speed at which a certain distance was covered. Since our paradigm made it possible to determine trajectory correction points for both change and go trials, we analyzed the velocities to and from this point for all trial types.

\section{Predictions}

Considering the relationships between the trajectory correction points in go and change trials, depending on the nature of the previous trial, the following patterns were expected.

Proactive inhibition (Fig. 2A) The trajectories for all three trial types should initially be biased toward the go location. The $x$ coordinate of the trajectory correction point should be close to zero (which represents the entrance point into the arena) for go trials following a go trial and for go trials following a change trial, but positive for change trials. The $y$-coordinate should be close to zero (which represents the entrance point into the arena) for go trials following a go trial and for go trials following a change trial, but greater than zero for change trials. Average velocities from entering to reaching the trajectory correction point, and in fact the overall velocities for the entire trial, should be slowest for change trials; velocities during go trials following a change trial should be close to the latencies for change trials; and velocities for go trials following a go trial would be expected to be the fastest. Once the correction point had been passed, velocities on change trials should slow down further, whereas those for go trials might become faster, though it would be expected that subjects would complete go trials following a go trial at a very fast speed throughout the trial.

Reactive inhibition (Fig. 2B) The trajectories for all three trial types should initially be biased toward the go location. The $x$ coordinate of the trajectory correction point should be close to zero (which represents the entrance point into the arena) for go trials following a go trial and for go trials following a change trial, but positive for change trials. The $y$-coordinate should be 
close to zero (which represents the entrance point into the arena) for go trials following a go trial and for go trials following a change trial, but greater than zero for change trials. Velocities up to the trajectory correction point should be equal for change trials and go trials. Once the correction point had been passed, velocities should slow down, with a greater decrease from initial velocities being observed in change trials than in either type of go trial.

Response repetition (Fig. 2C) The trajectories for go trials following a change trial and for change trials should initially be biased toward the incorrect location. The $x$-coordinate of the trajectory correction point in go trials following a go trial should be close to zero, whereas that of the trajectory correction point in go trials following a change trial should be negative, and that for a change trial should be positive. The $y$ coordinate should be close to zero for go trials following a go trial, but greater than zero (and of equal magnitude) for go trials following a change trial and for change trials. Velocities up to the trajectory correction point, as well as the overall velocities for the entire trial, should be equal for change trials and for go trials following a change, and the pigeons might additionally slow down once the correction point had been passed. Go trials following another go trial would be expected to be completed at a very fast speed throughout the trial.

No discrimination (Fig. 2D) The trajectories for all three trial types should initially run straight into the center of the arena and then converge on the correct location. The $x$-coordinates of the trajectory correction point should be close to zero in all trial types, but they might be biased toward the go location in change trials, and toward the change location in go trials, due to averaging, because the trajectory correction point would be close to zero for trials in which the pigeon approached the correct location first, but close to the incorrect location for trials in which that location was approached first. The $y$-coordinates of the trajectory correction point should be equal in all three trial types and should be greater than zero. Velocities up to the trajectory correction point, like overall velocities for the entire trial, should be equally long for all trial types, and it would be expected that the same speed would be maintained throughout a trial (with just a little increase in speed, if any at all, once a goal had been chosen).

\section{Results}

According to the specific predictions stated above, the most informative values for differentiating between the response strategies of interest are the coordinates of the trajectory correction points on the three types of trial (go following go, go following change, and change). We found no observable difference for any individual subject (see Supplementary Table 1) between the correction points for go trials following a go trial and for go trials following a change trial, which otherwise might have indicated that they had pursued a strategy of mere response repetition. The remaining strategies under investigation could be differentiated by assessing how much the trajectory correction points of the three trial types differed in terms of their distance from the start location and advancement toward the go location. For this purpose, each pigeon was ranked according to the average differences between the $y$-coordinate of its mean change-trial correction point and the $y$-coordinates of the mean correction points of the two types of go trials (the difference in the $y$-coordinate rather than the absolute distance between points was chosen in order to preserve the directionality of the distance-i.e., to be able to determine whether the change point of the go points were closer to the start location). In making this calculation, it emerged that not all subjects showed the anticipated bias to initially approach the go location before correcting their path if necessary; about an equal number of subjects expressed a reversed bias, approaching the change location first in all trials, and thus having to correct their path on go trials. It is noteworthy that such a bias during test did not correspond to any observed preference during habituation (Supplementary Table 1), potentially indicating that the pigeons only developed it after at least a few encounters with the arena during habituation and the first test session. For further analyses, the group was therefore split into five subgroups of approximately equal sizes, corresponding to whether the subjects expressed a strong, weak, or no apparent bias to initially approach the go or the change location. The individual trajectories and trajectory correction points, including latency information, are illustrated in Fig. 4.

All statistical analyses were performed using jamovi 0.9.5.8 (jamovi project, 2018). Analyses of variance (ANOVAs) were subject to Huynh-Feldt corrections where applicable, and post-hoc pairwise comparisons between groups were Tukey-corrected.

\section{Trajectory correction points}

As is shown in Table 1, the $x$-coordinates of the pigeons' average trajectory correction points differed significantly across the five bias groups $\left[F(4,33)=10.70, p<.001, \eta_{\mathrm{p}}{ }^{2}=\right.$ $.56]$. The same was true for the $y$-coordinates $[F(4,33)=$ $13.40, p<.001, \eta_{\mathrm{p}}{ }^{2}=.62$; see Table 1]. Therefore, all subsequent analyses were carried out separately for each group.

Strong go bias For those subjects having a strong bias to approach the go location in all trials (Figs. 4 and 5, top row), the $x$-coordinates were comparable for the two types of go trials, which both differed significantly from the $x$-coordinate on change trials $\left[F(2,14)=32.60, p<.001, \eta_{\mathrm{p}}{ }^{2}=.82\right.$; pairwise 


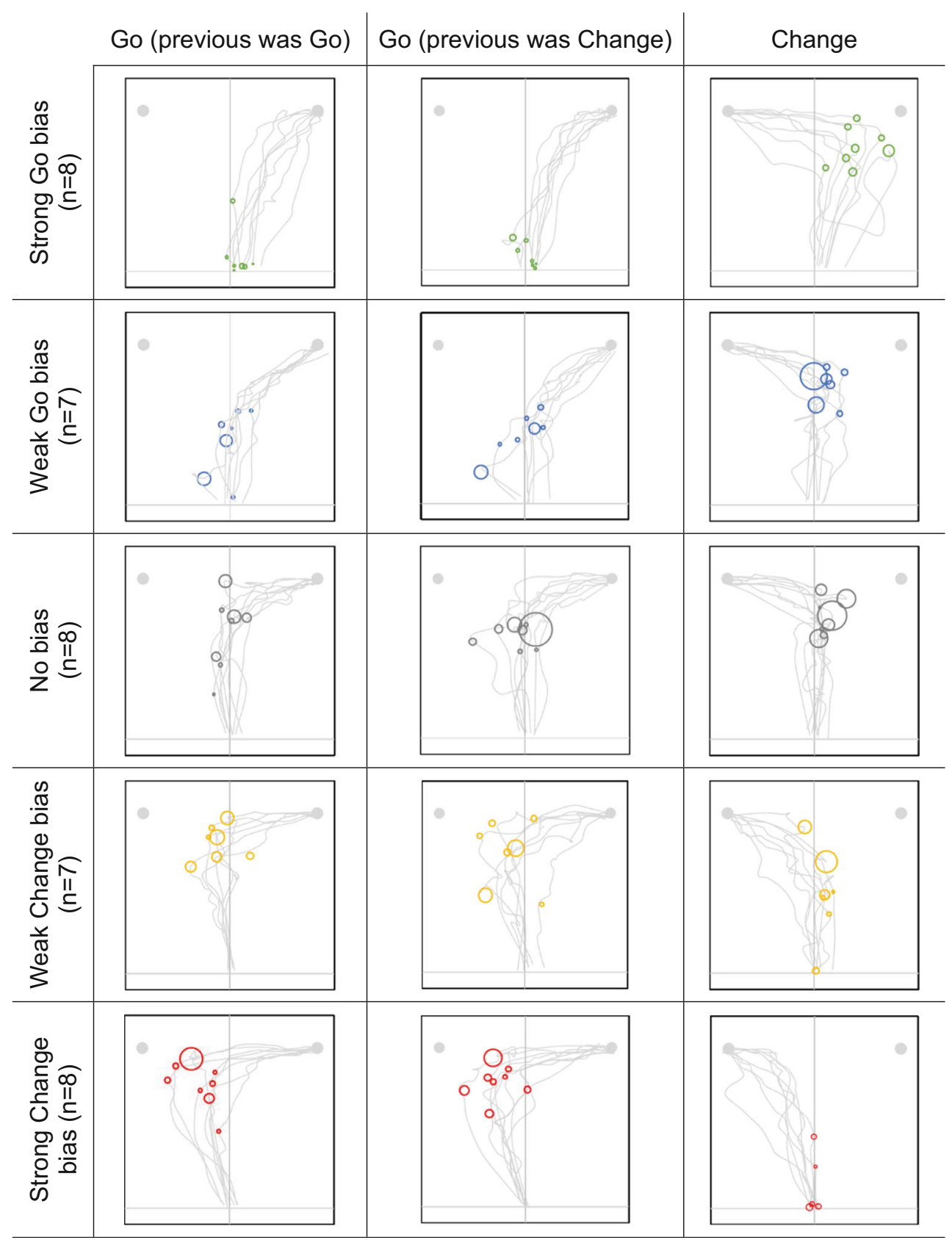

Fig. 4 Individual trajectories (gray lines) and trajectory correction points (open circles) of pigeons showing a strong, weak, or no bias toward approaching either the go or the change location, in go trials following a go trial, go trials following a change trial, and change trials. The diameter of each circle corresponds to the latency to reach the

comparison between go following go and go following change: $t(14)=1.47, p=.33$; comparison of either go trial type with change: $t(14)>4.14, p<.001]$. The $x$-coordinates of go trials following a go trial and of change trials were significantly greater than zero, indicating that the pigeons were closer to the go location than to the change location when they started approaching the correct location. In contrast, the $x$ coordinates of go trials following a change trial were not significantly different from zero [go following go: $t(7)=2.67, p=$ correction point upon entering the arena at $(0,0)$, where bigger circles indicate longer latencies. The gray, filled circles indicate the locations of the food wells; in go trials, the reinforced location was at $(1,1)$; in change trials, it was at $(-1,1)$.

.032 ; go following change: $t(7)=1.06, p=.32$; change: $t(7)=$ $5.98, p<.001]$. Likewise, $y$-coordinates differed significantly between change trials and either type of go trial, but were comparable between the two types of go trial $[F(2,14)=$ $135.00, p<.001, \eta_{\mathrm{p}}{ }^{2}=.95$; pairwise comparison between go following go and go following change: $t(14)=0.18, p=$ .98; comparison of either go trial type with change: $t(14)>$ $14.11, p<.001]$. The $y$-coordinates of go trials following a change trial and of change trials differed significantly from 
Table 1 Average $x$ - and $y$-coordinates of the trajectory correction point, with average velocities before and after reaching this point, for pigeons showing a strong, weak, or no bias toward approaching either the go location or the change location, in go trials following a go trial, go trials following a change trial, and change trials

\begin{tabular}{|c|c|c|c|c|c|}
\hline & & \multicolumn{2}{|c|}{ Trajectory Correction Point } & \multicolumn{2}{|l|}{ Velocity $(\mathrm{cm} / \mathrm{s})$} \\
\hline & & $x$ & $y$ & $\begin{array}{l}\text { Entrance to Trajectory } \\
\text { Correction Point }\end{array}$ & $\begin{array}{l}\text { Trajectory Correction } \\
\text { Point to Goal Location }\end{array}$ \\
\hline \multirow[t]{3}{*}{ Strong Go Bias } & Go (previous go) & 0.13 & 0.09 & 13.49 & 11.39 \\
\hline & Go (previous change) & 0.04 & 0.08 & 18.62 & 13.13 \\
\hline & Change & 0.49 & 0.77 & 15.51 & 6.86 \\
\hline \multirow[t]{3}{*}{ Weak Go Bias } & Go (previous go) & -0.01 & 0.40 & 20.60 & 7.74 \\
\hline & Go (previous change) & -0.05 & 0.44 & 23.31 & 6.90 \\
\hline & Change & 0.16 & 0.75 & 8.48 & 5.73 \\
\hline \multirow[t]{3}{*}{ No Bias } & Go (previous go) & -0.04 & 0.66 & 16.18 & 4.60 \\
\hline & Go (previous change) & -0.10 & 0.64 & 19.50 & 5.84 \\
\hline & Change & 0.14 & 0.76 & 7.97 & 3.95 \\
\hline \multirow[t]{3}{*}{ Weak Change Bias } & Go (previous go) & -0.14 & 0.82 & 10.38 & 5.48 \\
\hline & Go (previous change) & -0.20 & 0.74 & 10.32 & 5.25 \\
\hline & Change & 0.10 & 0.50 & 13.60 & 6.36 \\
\hline \multirow[t]{3}{*}{ Strong Change Bias } & Go (previous go) & -0.35 & 0.77 & 21.17 & 7.33 \\
\hline & Go (previous change) & -0.34 & 0.78 & 15.12 & 5.34 \\
\hline & Change & 0.00 & 0.10 & 14.89 & 12.40 \\
\hline
\end{tabular}

zero, indicating that the pigeons had moved a considerable distance from the start location into the arena at the point of correcting their path. In contrast, the $y$-coordinates of go trials following a go trial did not differ from zero [go following go: $t(7)=1.78, p=.12$; go following change: $t(7)=3.06, p=.018$; change: $t(7)=18.41, p<.001]$. In relation to the above predictions, this pattern suggests mental goal adjustments reflecting proactive response inhibition.

Weak go bias The $x$-coordinates of those pigeons that showed a weak bias toward the go location (Figs. 4 and 5, second row) were significantly different between go trials following a change trial and change trials but not between go trials following a go and any other trial type $\left[F(2,12)=5.00, p=.030, \eta_{\mathrm{p}}{ }^{2}\right.$ $=.45$; pairwise comparison between go following go and go following change: $t(12)=0.61, p=.82$, comparison between go following go and change: $t(12)=2.38, p=.083$; comparison between go following change and change: $t(12)=2.99, p$ $=.028]$. The $x$-coordinates of either type of go trial did not differ significantly from zero, whereas that of change trials was significantly greater than zero, indicating that these pigeons were closer to the go location than to the change location when finally starting to approach the correct change location [go following go: $t(6)=0.14, p=.89$; go following change: $t(6)=0.53, p=.62$; change: $t(6)=3.35, p=.016]$. The $y$-coordinates differed significantly between change trials and either type of go trial, but were comparable between the two types of go trial $\left[F(2,12)=22.70, p<.001, \eta_{\mathrm{p}}{ }^{2}=.79\right.$; pairwise comparison between go following go and go following change: $t(12)=0.73, p=.75$; comparison of either go trial type with change: $t(12)>5.44, p<.001]$. The $y$-coordinates of all three trial types differed significantly from zero, showing that the pigeons had considerably moved into the arena before correcting their paths [go following go: $t(6)=5.01, p=.002$; go following change: $t(6)=8.75, p<.001$; change: $t(6)=$ $18.04, p<.001]$. Taken together, the observed pattern provides some evidence for a reactive-inhibition account.

No bias Regarding those subjects that were labeled as having no distinct bias to initially approach either location (Figs. 4 and 5 , third row), the $x$-coordinates of go trials following a change trial and change trials were significantly different; go trials following a go did not differ from go trials following a change but were marginally different from change trials $[F(2$, 14) $=6.76, p=.017, \eta_{\mathrm{p}}{ }^{2}=.49$; pairwise comparison between go following go and go following change: $t(14)=0.96, p=$ .61 ; comparison between go following go and change: $t(14)=$ $2.59, p=.052$; comparison between go following change and change: $t(14)=3.55, p=.008]$. The $x$-coordinates of either type of go trial did not differ significantly from zero, whereas that of change trials was significantly greater, again indicating that the pigeons were closer to the go location than to the change location when they started to correct their path toward the change location [go following go: $t(7)=0.93, p=.38$; go following change: $t(7)=1.24, p=.25$; change: $t(7)=3.68, p=$ $.008]$. We found no significant difference between the three $y$ - 
coordinates $\left[F(2,14)=2.12, p=.18, \eta_{\mathrm{p}}^{2}=.23\right]$. The $y$-coordinates of all three trial types were significantly different from zero, indicating that the pigeons had considerably moved into the arena before correcting their paths [go following go: $t(7)=$ 8.31, $p<.001$; go following change: $t(7)=26.25, p<.001$; change: $t(7)=19.73, p<.001]$. The overall pattern could point toward a response repetition or a nondiscriminative approach, but evidence for either is weak.

Weak change bias The $x$-coordinates of those pigeons that showed a weak bias to initially walk toward the change location (Figs. 4 and 5, fourth row) were significantly different between go trials following a change trial and change trials but only marginally so between go trials following a go and change trials $\left[F(2,12)=5.39, p=.044, \eta_{\mathrm{p}}{ }^{2}=.47\right.$; pairwise comparison between go following go and go following change: $t(12)=0.58, p=.84$; comparison between go following go and change: $t(12)=2.51, p=.066$; comparison between go following change and change: $t(12)=3.09, p=$ .024]. The $x$-coordinates of either type of go trial did not differ from zero, that of change trials was marginally greater [go following go: $t(6)=1.82, p=.12$; go following change: $t(6)$ $=1.87, p=.11$; change: $t(6)=2.28, p=.062]$. The $y$-coordinates differed significantly between change trials and either type of go trial, but were comparable between the two types of go trial $\left[F(2,12)=8.57, p=.012, \eta_{\mathrm{p}}{ }^{2}=.59\right.$; pairwise comparison between go following go and go following change: $t(12)=0.92, p=.64$; comparison of either go trial type with change: $t(12)>3.04, p<.026]$. The $y$-coordinates of all three trial types differed significantly from zero, showing that the pigeons had considerably moved into the arena before correcting their paths [go following go: $t(6)=19.84, p<$ .001 ; go following change: $t(6)=9.35, p<.001$; change: $t(6)=4.76, p=.003]$. Taken together, and considering the initial bias toward the change location, the observed pattern provides some evidence for a reactive-inhibition account.

Strong change bias For those subjects with a strong bias toward the change location (Figs. 4 and 5, bottom row), $x$-coordinates were comparable for the two types of go trials, which both differed significantly from the $x$-coordinate of change trials $\left[F(2,14)=15.20, p<.001, \eta_{\mathrm{p}}^{2}=.69\right.$; pairwise comparison between go following go and go following change: $t(14)$ $=0.23, p=.97$; comparison of either go trial type with change: $t(14)>4.66, p<.001]$. The $x$-coordinates of either type of go trial were significantly smaller than zero-indicating that the pigeons were closer to the change location than to the go location when they started approaching the correct go location - whereas that of change trials was not different from zero [go following go: $t(7)=4.57, p=.003$; go following change: $t(7)=4.45, p=.003$; change: $t(7)=0.10, p=.93$ ] Likewise, the $y$-coordinates differed significantly between change trials and either type of go trial, but they were comparable between the two types of go trial $[F(2,14)=$ $55.50, p<.001, \eta_{\mathrm{p}}{ }^{2}=.89$; pairwise comparison between go following go and go following change: $t(14)=0.25, p=.97$; comparison of either go trial type with change: $t(14)>8.99, p$ $<.001]$. The $y$-coordinates of either type of go trial differed significantly from zero, whereas the $y$-coordinate of change trials did not [go following go: $t(7)=15.39, p<.001$; go following change: $t(7)=20.74, p<.001$; change: $t(7)=$ $1.74, p=.13]$. Considering the strong initial bias to approach the change location, this pattern is indicative of reactive inhibition.

\section{Velocities}

We focused on median rather than mean latencies when computing velocity, to avoid a misrepresentation of average speed due to the often atypically long latencies during the first trials of each session. Figure 4 presents each individual's average trajectories (gray lines) and median latencies (open circles), from which velocities were computed. Overall, velocities (Table 1; Fig. 5, right column) did not differ significantly between bias groups $\left[F(4,33)=1.52, p=.22, \eta_{\mathrm{p}}^{2}=.16\right]$. However, to preserve symmetry with the analyses above, the subsequent analyses were carried out for each group separately.

Strong go bias For those subjects having a strong bias to approach the go location in all trials (Fig. 5, top row), velocities did not differ significantly between trial types overall $\left[F(2,14)=2.63, p=.14, \eta_{\mathrm{p}}{ }^{2}=.27\right]$. However, velocities did decrease significantly after subjects reached the trajectory correction point $\left[F(1,7)=9.32, p=.019, \eta_{\mathrm{p}}{ }^{2}=.57\right]$. Despite the lack of a clear interaction effect between trial type and velocity before or after reaching the correction point, pairwise comparisons showed that the only statistically significant decrease occurred in change trials $\left[F(2,14)=3.54, p=.057, \eta_{\mathrm{p}}{ }^{2}=\right.$ .34; comparison of pre- and postcorrection velocities for go following go: $t(7)=0.92, p=.94$; for go following change; $t(7)=2.41, p=.21$; for change: $t(7)=3.81, p=.017]$. This pattern fits our prediction for reactive inhibition, in that the subjects seemed to react to a change in contingencies rather than to anticipate it, slowing down during change trials after adjusting their paths. The previous trial did not affect behavior significantly.

Weak go bias Those pigeons that expressed a weaker bias toward the go location (Fig. 5, second row) showed higher velocities during either type of go trial than during change trials $\left[F(2,12)=8.05, p=.010, \eta_{\mathrm{p}}{ }^{2}=.57\right.$; pairwise comparison between go following go and go following change: $t(12)$ $=0.43, p=.91$; comparison between go following go and change: $t(12)=3.24, p=.018$; comparison between go following ch 


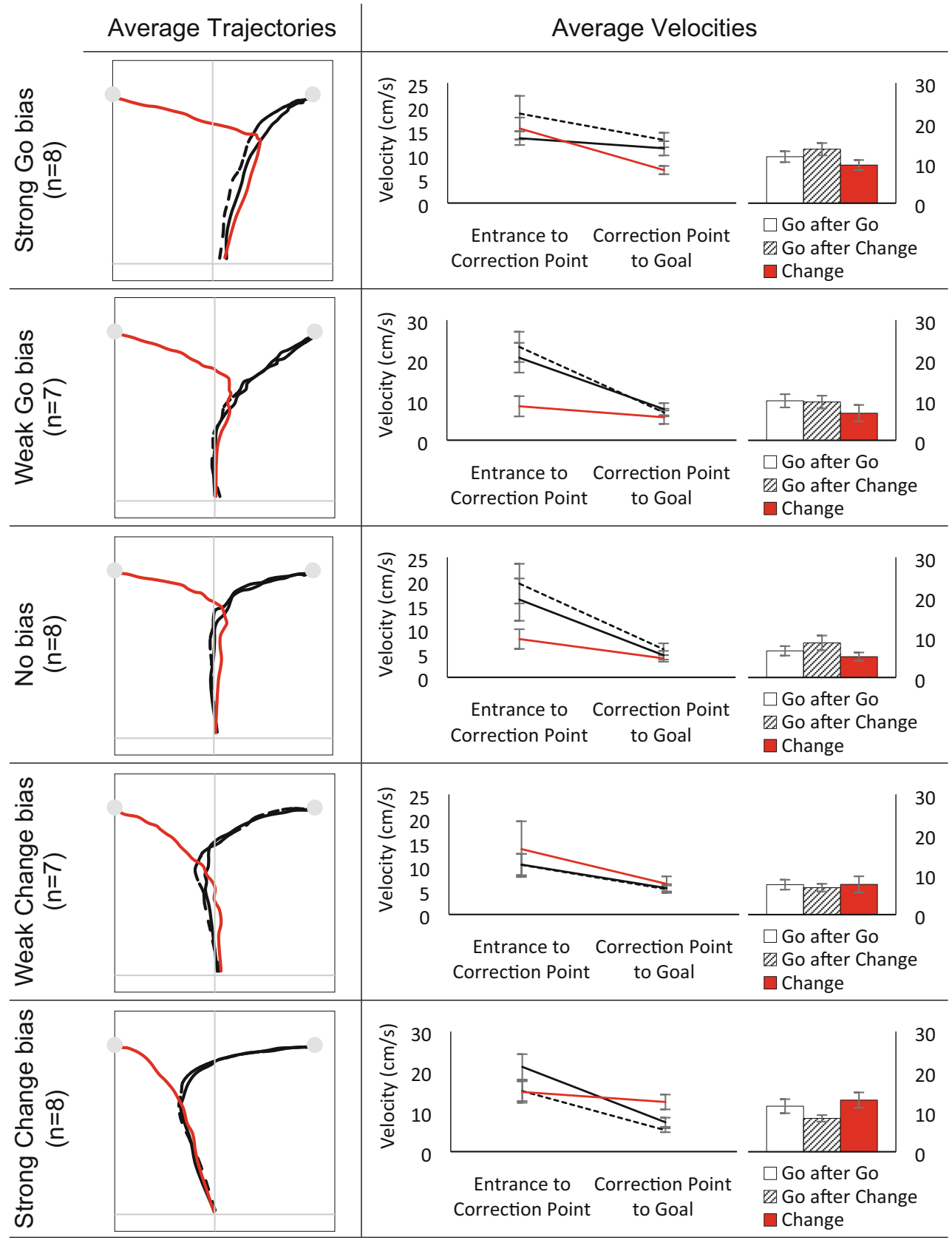

Fig. 5 Left column: Averaged trajectories per group and trial type: Black solid lines, go trials following a go trial; black dotted lines, go trials following a change trial; color lines, change trials. Note that on go trials, the correct location was on the right; on change trials, it was on the left. Right column: Average velocities to complete a trial, per group and trial type (measured from entering the arena to reaching the trajectory correction point and from the correction point to reaching the correct location). ange and change: $t(12)=3.67, p=.008]$. Furthermore, velocities decreased after subjects reached the trajectory correction point $\left[F(1,6)=22.41, p=.003, \eta_{\mathrm{p}}{ }^{2}=.79\right]$. We observed a significant interaction effect between the two factors, as the reduction in velocity was only significant for go trials, but not for change trials $\left[F(2,12)=9.35, p=.004, \eta_{\mathrm{p}}{ }^{2}=.61\right.$; comparison of pre- and postcorrection velocities for go following go: $t(6)=4.37, p=.007$; for go following change: $t(6)=5.57$, $p<.001$; for change: $t(6)=0.93, p=.93$ ] Furthermore, change-trial velocity was only significantly lower than gotrial velocity before the correction point was reached, but not after [pairwise comparison of precorrection velocity in change as compared with go trials following a go or a change trial: both $t(12)>4.45, p<.002$; comparison of go following go and go following change: $t(12)=0.99, p=.92$; comparison of postcorrection velocities between trials: all $p \mathrm{~s}>.98]$. This pattern does not directly match our predictions for reactive inhibition (as we assumed that any slowing in velocities 
during change trials would occur primarily once the correction point had been reached), but it shows that these subjects were already considerably slower during change than during go trials, even before reaching the trajectory correction point.

No bias For subjects without a distinct bias toward either location (Fig. 5, third row), velocities for change trials were marginally significantly different from those for go trials following a change trial $\left[F(2,14)=3.92, p=.062, \eta_{\mathrm{p}}{ }^{2}=.36\right]$, and velocities reduced significantly from pre- to postcorrection $\left[F(1,7)=21.89, p=.002, \eta_{\mathrm{p}}{ }^{2}=.76\right]$. The interaction effect was also marginally significant, as the reduction in velocity was only found in go but not in change trials $[F(2,14)=3.79$, $p=.066, \eta_{\mathrm{p}}{ }^{2}=.35$; comparison of pre- and postcorrection velocities for go following go: $t(7)=3.89, p=.011$; for go following change: $t(7)=4.59, p=.002$; for change: $t(7)=$ $1.35, p=.76]$. Likewise, lower velocity in change than in go trials was only shown in precorrection velocities, but not postcorrection [pairwise comparison of precorrection velocities in change vs. go trials following a change trial: $t(14)=$ $3.78, p=.010$; comparisons of change vs. go following go: $t(14)=2.69, p=.11$; comparison of go following go vs. go following change: $t(14)=1.09, p=.88$; comparison of postcorrection velocities between trials: all $p \mathrm{~s}>$.99]. As above, this pattern indicates that these pigeons moved slowly primarily during change trials, suggesting that even though their trajectories did not show an obvious bias toward the go location, these pigeons nonetheless faced more difficulties pursuing the goal during change trials.

Weak change bias Subjects expressing a weak bias toward the change location (Fig. 5, fourth row) did not show any significant differences between the velocities of different trial types $\left[F(2,12)=0.44, p=.58, \eta_{\mathrm{p}}{ }^{2}=.07\right]$. Velocities decreased marginally after subjects reached the trajectory correction point $\left[F(1,6)=4.20, p=.086, \eta_{\mathrm{p}}{ }^{2}=.41\right]$. There was no interaction effect between the two factors $[F(2,12)=0.44, p$ $\left.=.56, \eta_{\mathrm{p}}{ }^{2}=.07\right]$. This pattern fits the predictions regarding a nondiscriminative approach.

Strong change bias Finally, those pigeons that were strongly inclined to approach the change location first (Fig. 5, bottom row) showed marginal differences in overall velocities between trial types $\left[F(2,14)=3.18, p=.072, \eta_{\mathrm{p}}{ }^{2}=.31\right]$. More prominent, however, were the significant decrease in velocity after reaching the correction point $[F(1,7)=44.31, p<.001$, $\left.\eta_{\mathrm{p}}{ }^{2}=.86\right]$ and a significant interaction effect, showing that this decrease only occurred in go but not in change trials $[F(2,14)$ $=9.03, p=.003, \eta_{\mathrm{p}}{ }^{2}=.56$; comparison of pre- and postcorrection velocities for go following go: $t(7)=6.80, p$ $<.001$; for go following change: $t(7)=4.80, p=.001$; for change: $t(7)=1.23, p=.82]$. Furthermore, pairwise comparisons showed that velocities only differed marginally between trial types before the correction point was reached, whereas there was a significant difference between go trials following a change and change trials after that point was passed [pairwise comparison of precorrection velocity in go trials following a change trial vs. go trials following a change trial, $t(14)=2.77$, $p=.095$; comparisons of go following go vs. change, $t(14)=$ $2.87, p=.076$; comparison of go following change vs. change: $t(14)=0.10, p=1.0 ;$ comparison of postcorrection velocities in go following go and in go following change: $t(14)=0.91, p$ $=.94$; comparison of go following go vs. change: $t(14)=2.32$, $p=.22$; comparison of go following change vs. change: $t(14)$ $=3.23, p=.035]$. Taking into account the bias toward change of these subjects, this pattern fits the predictions of reactive inhibition. Although subjects tended to start go trial following a change trial at a somewhat lower speed than go trials following a go, this pattern indicates that they mostly reacted to a change in contingencies rather than anticipating it, slowing down during go trials after adjusting their paths.

\section{Discussion}

The ability to inhibit and correct one's actions quickly depending on changes in the environment seems essential to survival. Yet, it has been challenging to assess, or even experimentally quantify, the mental processes enabling response inhibition. The continuous inhibitory-control task presented in this study was designed to provide observable and measurable variables to achieve this. The paradigm required pigeons to approach a baited food location, a behavior that is highly prevalent in their natural behavioral repertoire and is executed quickly, and would thus require controlled inhibition in order to overcome (cf. Meier et al., 2017).

Pigeons have shown a good capacity to employ reactive inhibitory control in computerized experiments (Lea et al., 2019; Meier et al., 2018), and were thus a suitable study species to investigate whether this task could reliably identify and contrast inhibitory-control processes from less cognitively demanding strategies. Indeed, we observed a range of different approaches to this paradigm amongst our subjects. In an effort to determine whether the observed response patterns could provide insight into cognitive processes, we specified several potential strategies that might underlie performance in this task and sketched the expected trajectories as well as speed while approaching a location if each of these strategies was pursued.

First, controlled response inhibition can be characterized not only by a slowing in speed when behavior has to be adjusted to a sudden change, but also by the paths taken to pursue a goal: an initial movement toward a predisposed location, followed by a sharp curve toward an alternative location after the subject realizes that the initial location is unavailable (see Fig. 2A). During trials in which no such sudden 
change occurred and in which no path correction is necessary, however, trajectories approach the initially chosen location in a straight line from the starting point. Both the human participants in Verbruggen and McLaren's (2017) study and the pheasants tested by Meier et al. (2017) showed this pattern of behavior, as did many of the pigeons in this study (Fig. 5, left column), providing evidence that response inhibition was necessary to correct one's performance. Interestingly, despite the go location always being the initially available goal (and being reinforced twice as often), we observed an almost even split in our sample between subjects that initially approached the go location (Fig. 4, top two rows, "strong go bias" and "weak go bias") and those that set out toward the at this point visibly unavailable change location (Fig. 4, bottom two rows, "weak change bias" and "strong change bias"). This observation is the first indication that the pigeons' approach to the task might not fit the predicted patterns. The even split between pigeons that approached the go location first and those that primarily approached the change location suggests that, instead of the anticipated bias toward go, at least a portion of the pigeons might have always followed their own internal preference for one of the locations that was independent of the presented information. In such a case, the particular conditions of a trial will have had very little influence on the pigeons' initial behavior. For those pigeons that preferentially approached the go location, it is impossible to determine whether their bias was determined by an inherent preference or an attention to the task contingencies. However, either bias can be described by a common response patterns: Trajectories toward the preferred location were straight and the trajectory correction points were very close to the start location from which the pigeons entered the arena, whereas the paths toward the nonpreferred location were curved, with a correction point farther into the arena. Taken together, this indicates that the pigeons most likely started their trajectory with an automatic, non-information-driven, response and subsequently had to exert inhibitory control to overcome this bias and approach the correct location.

As we noted above, as this approach begins with a subject's internal preference for one particular location, it is unlikely that the initial trajectory or speed when approaching a goal were influenced by events on previous trials, which as we argue is an important signpost of an individual's ability to perform sophisticated inhibitory-control strategies - in particular its capacity to optimize responses by performing the mental goal adjustments indicative of proactive inhibitory control (Elchlepp et al., 2016; Verbruggen \& McLaren, 2017)-proposed by the mental goal adjustments account (Fig. 2A). However, the observed response patterns of the majority of our subjects provide coherent evidence for relying on reactive inhibitory control to solve the task (Fig. 5, top two rows, "strong go bias," "weak go bias," and bottom row, "strong change bias"; the subgroup "weak change bias," though expressing the predicted pattern in their trajectories, did not show the expected velocities as per the reactive inhibition account). Even though subjects did not show evidence of anticipating a potential change in contingencies, this form of inhibitory control allows subjects to quickly react if the initially approached location is not available by slowing down and altering their path. It has to be noted that, although we predicted that for reactive inhibition, velocities would be slower after than before reaching this trajectory correction point, the observed patterns (which matched this prediction) could also reflect a general slowing across the path rather than a change at the correction point. It is therefore possible that the pigeons started fast and progressively slowed down as a result of reactive control being progressively engaged. Only an examination of individual changes in speed across an entire trial would allow inferences about this possibility, and that could not be carried out in the present study.

As can be seen in Fig. 5, not all pigeons pursued their preferred goal with equal fervor. Those with the strongest bias (Fig. 5, top row, "strong go bias," and bottom row, "strong change bias") also demonstrated the fastest velocities, whereas those subjects with a less pronounced bias (Fig. 5, second row, "weak go bias," and fourth row, "weak change bias") were somewhat slower. It might have been the case that strongly biased subjects generally completed the movement toward their preferred location until they reached it and only approached the opposite location after verifying that the preferred location was inaccessible. In fact, any predictions based on latencies or velocities are unable to differentiate between such failed and successful inhibition, as in either case, latencies would be expected to be longer during change than during go trials and averaged velocities to be generally slower on change trials, when subjects need to slow down (or stop entirely) in order to be able to correct their current path. Thus, only an examination of individual trajectories can provide information about whether inhibition was achieved. Trajectory correction points very close to the incorrect goal location and sharp-angled trajectories on change trials (or, in the case of a reverse bias, go trials) speak to the assumption that the more strongly biased subjects among these two subgroups might indeed have faced difficulties inhibiting their preference. That leaves the question of whether those subjects with a less pronounced bias can be regarded as the truly successful "inhibitors." Some research suggests that human adults might possess a dual system of impulse control, consisting of a "cool" cognitive, self-regulatory system and a "hot" emotional, stimulus-driven system that defies cognitive control, with the balance between the two being determined by the emotional, developmental, and cognitive states of the individual (e.g., Hofmann, Friese, \& Strack, 2009; Metcalfe \& Mischel, 1999). Such a dual system might also exist for pigeons, with more strongly biased subjects being governed more by the hot system than are less-biased subjects. 
The performance of those pigeons that were grouped into the "weak bias" subgroups during our ranking procedure appear rather varied (see Fig. 4), so this issue should be explored further in future studies.

Second, the pigeons that showed no apparent bias toward either location also did not show a convincing pattern of employing inhibitory control to solve the task. An alternative strategy that might have guided behavior of these subjects could have been a tendency to return to the location that was rewarded in the previous trial. In this case, we would have expected greatly different trajectories for the two different types of go trials. This was not observed for any subject; most often, subjects took very similar paths toward the go location, regardless of whether that location had been reinforced in the previous trial (in the case of go trials following another go trial) or not (in the case of go trials following a Change trial). Just as above, we can therefore reasonably conclude that the response requirements of the previous trial had little influence on the pigeons' behavior.

Finally, we considered the potential response pattern that would emerge in the case that subjects did not discriminate between the two potential goal locations. Indeed, we were able to identify a subgroup of subjects that appeared to fall into this category (Fig. 5, third row, "no bias"). Their average trajectories led straight toward the center of the arena in all three types of trials, before turning toward the correct food location (Fig. 4, middle row). Thus, these pigeons moved into the arena without a clear overall preference for one or the other food location, perhaps due to hesitation to approach any location before the correct response requirements were known, or due to a tendency to randomly choose a location at the start of a trial and approach it regardless of the current requirements (which, when averaged out over multiple trials, would result in a straight line). A further point of note is that this subgroup of nondiscriminators also showed the lowest overall velocities when completing any trial, as compared to the other four groups, further highlighting their hesitant approach. Our paradigm relied on the assumption that pigeons would be quick to approach available food, because doing so is a highly prevalent natural behavior. However, we did not impose any negative consequences for approaching the incorrect location or for responding slowly; therefore, there was no implied demand to quickly readjust any incorrect responses, even though other subjects were clearly able to do so. Nonetheless, there seems to be more than meets the eye for these subjects regarding their ability (or apparent lack of ability) to discriminate between go and change trials: They proceeded to the trajectory correction point at the lowest speed during change trials - perhaps the perceived tippingover of the see-saw right after they entered the arena caused them to slow down further, without affecting their previously determined path.
In conclusion, the continuous response-inhibition paradigm used in this study has proven to be a valuable tool to assess behavioral strategies when faced with a task that can optimally be solved by employing inhibitory control. One of this paradigm's strong advantages in this regard is that it uses a subject's inherent drive to approach a food source, without the necessity to administer a large number of training sessions prior to the test. Although incorporating both trajectories and velocities proved difficult, the paradigm nonetheless provides several options to assess an individual's cognitive capacities in this regard, and the comparison between different measures of inhibitory control highlights that physical movement in space may add additional information about cognitive processes beyond measures based on speed. Pigeons are capable of applying a variety of cognitive approaches to the problem, including inhibitory control, although their capabilities might not be sufficient to express sophisticated control strategies such as the mental goal adjustments expressed by humans.

Acknowledgements This work was completed as part of C.M.'s PhD studies. Data from the experiment described in this article were presented at the meeting of the Comparative Cognition Society, held in Melbourne, Florida, in April 2019. We are grateful for comments and suggestions made by colleagues present at that meeting.

Open practices statement The data and materials for all experiments are available upon request from the first author and will be made available in a data repository prior to publication.

\section{Compliance with ethical standards}

Conflict of interest The authors declare that they have no conflict of interest

\section{References}

Ardila, A. (2008). On the evolutionary origins of executive functions. Brain and Cognition, 68, 92-99. https://doi.org/10.1016/j.bandc. 2008.03.003

Beuk, J., Beninger, R. J., \& Paré, M. (2014). Investigating a race model account of executive control in rats with the countermanding paradigm. Neuroscience, 263, 96-110. https://doi.org/10.1016/j. neuroscience.2014.01.014

Bissett, P. G., \& Logan, G. D. (2011). Balancing cognitive demands: Control adjustments in the stop-signal paradigm. Journal of Experimental Psychology: Learning, Memory, and Cognition, 37, 392-404. https://doi.org/10.1037/a0021800

Bissett, P. G., \& Logan, G. D. (2012). Post-stop-signal adjustments: Inhibition improves subsequent inhibition. Journal of Experimental Psychology: Learning, Memory, and Cognition, 38, 955-966. https://doi.org/10.1037/a0026778

Brown, D. (2009). Tracker video analysis and modeling tool (Version 4.94) [Computer software]. Retrieved from http://physlets.org/ tracker/.

Chan, R. C. K., Shum, D., Toulopoulou, T., \& Chen, E. Y. H. (2008). Assessment of executive functions: Review of instruments and identification of critical issues. Archives of Clinical Neuropsychology, 23, 201-216. https://doi.org/10.1016/j.acn.2007.08.010 
Coutlee, C. G., \& Huettel, S. A. (2012). The functional neuroanatomy of decision making: Prefrontal control of thought and action. Brain Research, 1428, 3-12. https://doi.org/10.1016/j.brainres.2011.05. 053

Eagle, D. M., \& Robbins, T. W. (2003). Lesions of the medial prefrontal cortex or nucleus accumbens core do not impair inhibitory control in rats performing a stop-signal reaction time task. Behavioural Brain Research, 146, 131-144. https://doi.org/10.1016/j.bbr.2003.09.022

Elchlepp, H., Lavric, A., Chambers, C. D., \& Verbruggen, F. (2016). Proactive inhibitory control: A general biasing account. Cognitive Psychology, 86, 27-61. https://doi.org/10.1016/j.cogpsych.2016.01. 004

Fagnani, J., Barrera, G., Carballo, F., \& Bentosela, M. (2016). Is previous experience important for inhibitory control? A comparison between shelter and pet dogs in A-not-B and cylinder tasks. Animal Cognition, 19, 1165-1172. https://doi.org/10.1007/s10071-0161024-Z

Genest, C. (1992). Vincentization revisited. Annals of Statistics, 20, 1137-1142. Retrieved from http://www.jstor.org/stable/2242003

Hofmann, W., Friese, M., \& Strack, F. (2009). Impulse and self-control from a dual-systems perspective. Perspectives on Psychological Science, 4, 162-176.

jamovi project. (2018). jamovi (Version 0.8) [Computer Software].

Jurado, M. B., \& Rosselli, M. (2007). The elusive nature of executive functions: A review of our current understanding. Neuropsychology Review, 17, 213-233. https://doi.org/10.1007/s11065-007-9040-z

Kabadayi, C., Bobrowicz, K., \& Osvath, M. (2018). The detour paradigm in animal cognition. Animal Cognition, 21, 21-35. https://doi.org/ 10.1007/s10071-017-1152-0

Knolle, F., Goncalves, R. P., Davies, E. L., Duff, A. R., \& Morton, J. A. (2019). Response-inhibition during problem solving in sheep (Ovis aries). International Journal of Comparative Psychology. Preprint retrieved from https://escholarship.org/content/qt5074g4gq/ qt5074g4gq.pdf

Knolle, F., McBride, S. D., Stewart, J. E., Goncalves, R. P., \& Morton, A. J. (2017). A stop-signal task for sheep: introduction and validation of a direct measure for the stop-signal reaction time. Animal Cognition, 20, 615-626. https://doi.org/10.1007/s10071-017-10857

Lea, S. E. G., Chow, P. K. Y., Meier, C., McLaren, I. P. L., \& Verbruggen, F. (2019). Pigeons' performance in a tracking stop-signal procedure is consistent with the independent horse-race model. Journal of Experimental Psychology: Animal Learning and Cognition, 45, 464-473. https://doi.org/10.1037/xan0000219

Lucon-Xiccato, T., Gatto, E., \& Bisazza, A. (2017). Fish perform like mammals and birds in inhibitory motor control tasks. Scientific Reports, 7, 13144. https://doi.org/10.1038/s41598-017-13447-4

Marshall-Pescini, S., Virányi, Z., \& Range, F. (2015). The effect of domestication on inhibitory control: Wolves and dogs compared. PLoS ONE, 10, e0118469. https://doi.org/10.1371/journal.pone.0118469

Meier, C., Lea, S. E. G., \& McLaren, I. P. L. (2018). Pigeons in control of their actions: Learning and performance in stop-signal and changesignal tasks. Journal of Experimental Psychology: Animal Learning and Cognition, 44, 82-94. https://doi.org/10.1037/xan0000155
Meier, C., Pant, S. R., van Horik, J. O., Laker, P. R., Langley, E. J. G., Whiteside, M. A., ... Madden, J. R. (2017). A novel continuous inhibitory-control task: Variation in individual performance by young pheasants (Phasianus colchicus). Animal Cognition, 20, 1035-1047. https://doi.org/10.1007/s10071-017-1120-8

Metcalfe, J., \& Mischel, W. (1999). A hot/cool-system analysis of delay of gratification: Dynamics of willpower. Psychological Review, 106, 3-19. https://doi.org/10.1037/0033-295X.106.1.3

Morgan, M. J. (1974). Effects of random reinforcement sequences. Journal of the Experimental Analysis of Behavior, 22, 301-310.

Ratcliff, R. (1979). Group reaction time distributions and an analysis of distribution statistics. Psychological Bulletin, 86, 446-461. https:// doi.org/10.1037/0033-2909.86.3.446

Rouder, J. N., \& Speckman, P. L. (2004). An evaluation of the Vincentizing method of forming group-level response time distributions. Psychonomic Bulletin \& Review, 11, 419-427. https://doi.org/ 10.3758/BF03196589

Santacà, M., Busatta, M., Savașç1, B. B., Lucon-Xiccato, T., \& Bisazza, A. (2019). The effect of experience and olfactory cue in an inhibitory control task in guppies, Poecilia reticulata. Animal Behaviour, 151, 1-7. https://doi.org/10.1016/j.anbehav.2019.03.003

Suchy, Y. (2009). Executive functioning: Overview, assessment, and research issues for non-neuropsychologists. Annals of Behavioral Medicine, 37, 106-116. https://doi.org/10.1007/s12160-009-9097-4

Verbruggen, F., \& Logan, G. D. (2009). Models of response inhibition in the stop-signal and stop-change paradigms. Neuroscience and Biobehavioral Reviews, 33, 647-661. https://doi.org/10.1016/j. neubiorev.2008.08.014

Verbruggen, F., \& Logan, G. D. (2015). Evidence for capacity sharing when stopping. Cognition, 142, 81-95. https://doi.org/10.1016/j. cognition.2015.05.014

Verbruggen, F., McLaren, I. P. L., \& Chambers, C. D. (2014). Banishing the control homunculi in studies of action control and behavior change. Perspectives on Psychological Science, 9, 497-524. https://doi.org/10.1177/1745691614526414

Verbruggen, F., \& McLaren, R. (2017). Development of between-trial response strategy adjustments in a continuous action control task: A cross-sectional study. Journal of Experimental Child Psychology, 162, 39-57. https://doi.org/10.1016/j.jecp.2017.05.002

Verbruggen, F., Schneider, D. W., \& Logan, G. D. (2008). How to stop and change a response: The role of goal activation in multitasking. Journal of Experimental Psychology: Human Perception and Performance, 34, 1212-1228. https://doi.org/10.1037/0096-1523. 34.5.1212

Vernouillet, A. A. A., Stiles, L. R., McCausland, J. A., \& Kelly, D. M. (2018). Individual performance across motoric self-regulation tasks are not correlated for pet dogs. Learning \& Behavior, 46, 522-536. https://doi.org/10.3758/s13420-018-0354-x

Vincent, S. B. (1912). The function of the vibrissae in the behavior of the white rat. Animal Behavior Monographs, 1, 84.

Publisher's note Springer Nature remains neutral with regard to jurisdictional claims in published maps and institutional affiliations. 